\title{
Correction to: Detection of acute rib fractures on CT images with convolutional neural networks: effect of location and type of fracture and reader's experience
}

\author{
Minako Azuma ${ }^{1} \cdot$ Hiroshi Nakada ${ }^{1} \cdot$ Mizuki Takei $^{2} \cdot K^{K e i g o ~ N a k a m u r a}{ }^{2} \cdot$ Shigehiko Katsuragawa ${ }^{3} \cdot$ \\ Norihiro Shinkawa ${ }^{1} \cdot$ Tamasa Terada $^{1} \cdot$ Rie Masuda $^{1}$ - Youhei Hattori ${ }^{1} \cdot$ Takakazu Ide $^{1}$ - Aya Kimura ${ }^{1}$. \\ Mei Shimomura ${ }^{1} \cdot$ Masatsugu Kawano $^{1} \cdot$ Kengo Matsumura $^{1} \cdot$ Takayuki Meiri $^{1} \cdot$ Hidenobu Ochiai $^{4} \cdot$ Toshinori Hirai $^{5}$
}

Published online: 15 December 2021

(c) American Society of Emergency Radiology 2021

Correction to: Emergency Radiology (2021)

https://doi.org/10.1007/s10140-021-02000-6

Originally, the article was published with inverted name of the Author Aya Kimura, incorrect Fig. 3 caption and incomplete references 11 to 12 . We have now corrected the errors accordingly.

The original article has been corrected.

Publisher's note Springer Nature remains neutral with regard to jurisdictional claims in published maps and institutional affiliations.

The original article can be found online at https://doi.org/10.1007/ s10140-021-02000-6.

Minako Azuma

minako_azuma@med.miyazaki-u.ac.jp

1 Department of Radiology, Faculty of Medicine, University of Miyazaki, 5200 Kihara, Kiyotake, Miyazaki 889-1692, Japan

2 FUJIFILM Corporation, Tokyo, Japan

3 Department of Radiological Sciences, Teikyo University, Fukuoka, Japan

4 Center for Emergency and Critical Care Medicine, Faculty of Medicine, University of Miyazaki, Miyazaki, Japan

5 Department of Diagnostic Radiology, Faculty of Life Sciences, Kumamoto University, Kumamoto, Japan 